\title{
Research on the Frequency Domain Equalization of Single Carrier system applied in $\mathrm{HF}$ communication
}

\author{
$\mathrm{Lu} \mathrm{Si}$ \\ Digital Engineering Center \\ Communication University of China \\ Beijing, China \\ silu@cuc.edu.cn
}

\author{
Xinle $\mathrm{Yu}$ \\ Digital Engineering Center \\ Communication University of China \\ Beijing, China \\ yuxinle@cuc.edu.cn
}

\begin{abstract}
The application of frequency domain equalization technique makes single carrier modulation a valuable alternative resisting the ISI caused by the multipath channel in the broadband HF communication. This paper does a research on the principle and characteristics of Single Carrier systems with Frequency Domain Equalization (SCFDE), and gives the performance of SC-FDE based on different equalization schemes under multipath channel.
\end{abstract}

Keywords-HF channel; ISI; Single carrier; FDE; BER

\section{INTRODUCTION}

As we all know, HF communication is the only way to realize the global non relay communication. It is widely used in aviation and military field. However, the HF channel is multipath channel, and it has the selective fading of frequency, which seriously affect the reliability of communication. The traditional occupied bandwidth is mainly about $3 \mathrm{KHz}$, which can not meet the requirements and limits the application of the modern $\mathrm{HF}$ communication.

It is difficult to raise the bandwidth and data rate because of the high complexity of time domain equalizer(TDE). SC-FDE (Single Carrier Frenquency Domain Equalization)[1] and OFDM (Orthogonal Frequency Division Multiplexing) are both technologys who can effectively resist the ISI(Inter Symbol Interference) caused by the multipath channel, in which SC-FDE is more widly eyed by people owing to its advantages of low PARR(Peak to average Ratio Requirements) and less sensitive to the frequency offset compared to OFDM system. Now the SC-FDE has already been acceptted by the IEEE $802.16 \mathrm{a}$ as the recommended standard used in physical layer. In addition , corresponding to SC-FDE, multiple access(MA) technique is identified as one of the uplink transmission techniques for LTE physical layer by 3GPP, to resolve the problem of large transmission power of OFDM terminal.

The paper does a research on the FDE techniques of SC systems based on the MATLAB simulator[2].

\section{THE PRINCIPLE AND STRUCTURE OF SC-FDE}

SC-FDE and OFDM are closed in the system structure, and both have the similar means of signal processing that in each of these frequency domain systems. Fig .1 gives the system flow of SC-FDE, and the concrete digital signal processing is described as follows: the sending part firstly adopts technology of bit-to-symbol mapping, then adding unique words (UW) as cyclic prefix(CP) to data framing, at last data frames are send through the channel to transmit after shaped filtering. At the receiving part, the UW in each data block is used to estimate the characteristics of the channel and get the equalizing coefficients. To the data received, processing of FFT is used to get the frequency characteristics. The equalizing coefficients are multiplied by the corresponding data in the sub channels, then the data is converted to time domain by the IFFT module and UW is removed. At last the de-mapping and decision module realize the function of de-mapping and decision.

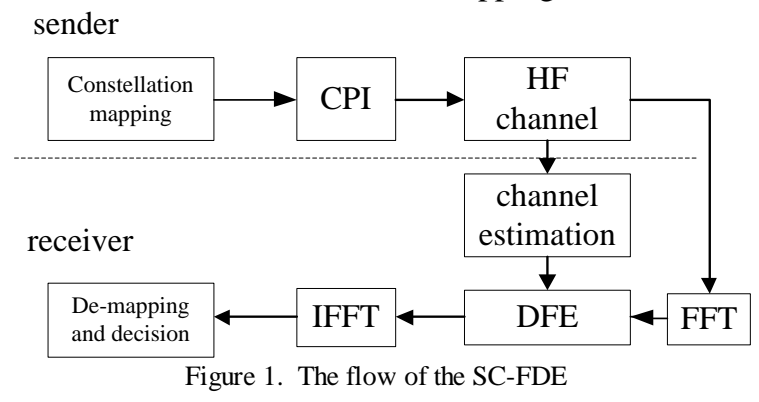

\section{FREQUENCY DOMAIN EQUALIZATION}

\section{A. The Flow of FDE}

The precondition of adopting the FDE is the data block transmission by adding UW to realize the conversion from linear convolution to cycle convolution. Meanwhile the channel characteristics is known. The basic process of SCFDE is to estimate the channel characteristics at first, and then the equalizer produces the coefficients $\mathrm{W}(\mathrm{k})$ which have the inverse characteristics, so it can resist the ISI caused by the multipath channel. Then, in each sub channel, the received data is multiplied by the equalization coefficient to compensate for the channel characteristics. 
Also We can consider it that the equalizer is used to convergent the extended energy of transmitting symbol to its own time slot. It is equal to insert a filter to make it with the sub channel to have constant amplitude and liner phase [3].

Fig .2 shows the flow of FDE, in which $\mathrm{y}(\mathrm{k})$ are symbols received and $\mathrm{Y}(\mathrm{k})$ are the in the frequency domain. $\mathrm{Z}(\mathrm{k})$ are the multiplication of $\mathrm{Y}(\mathrm{k})$ and $\mathrm{W}(\mathrm{k})$, and $\mathrm{z}(\mathrm{k})$ are $\mathrm{Z}(\mathrm{k}) \mathrm{by}$ IFFT.

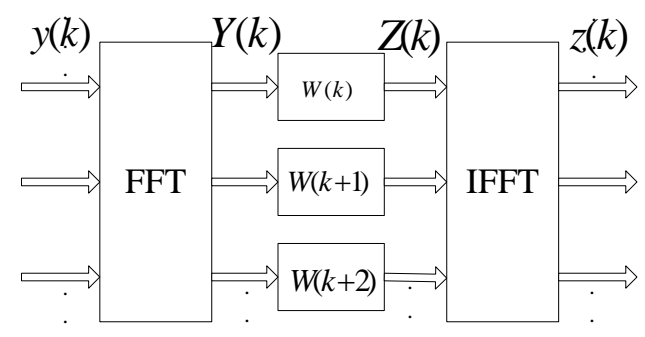

Figure 2. The flow of the FDE

\section{B. The Classification of Schemes}

The technology of FDE contains liner equalization and nonlinear equalization, and it is divided by the structure of equalizer, that is whether the output is used for feedback. Liner equalization includes zero forcing $(Z F)$ and the scheme based on Minimum Mean Square Error criterion (MMSE). The equalizing coefficients with ZF are :

And using MMSE are:

$$
W(z)=\frac{1}{H(z)},(k=0,1 \ldots n-1)
$$

$$
W(k)=\frac{H^{*}(k) \sigma_{S}^{2}}{|H(k)|^{2} \sigma_{S}^{2}+\sigma_{N}^{2}}=\frac{H^{*}(k)}{|H(k)|^{2}+\frac{1}{S N R}}
$$

The MMSE scheme considers the influence of noise and channel at the same time, so it has better performance than ZF especially in the condition of low SNR, while the ZF has a less calculation[4].

Nonlinear equalization is mainly the decision feedback equalization with the shortcoming of error propagation.N.Benvenuto[5] first introduced a hybrid decision feedback equalization scheme for time and frequency hybrid structures, called H-DFE. N.Benvenuto [6] then proposed a complete frequency domain decision feedback equalization scheme, and introduced the iterative structure, which is the IBDFE.Its performance is significantly better than the MMSE and H-DFE, under the condition of ideal channel estimation. But this scheme needs to know the signal statistics, and each iteration needs to be re calculated, so that the complexity is quite high. Considering the limit of space,the formulas of H-DFE and IBDFE are not listed in this paper.For short, H-DFE is referred to as DFE in the post text.

This paper simulate the SC-FDE adopting the liner equalization including $\mathrm{ZF}$ and MMSE, and the nonlinear equalization including DFE and IBDFE, based on the MATLAB simulator.

\section{SimUlation}

\section{A. Design of the Frame Structure}

In this simulation, adopts the single UW frame structure. UW at the head of data block is used as guard interval similar to the CP inverted in OFDM data blocks[7], while UW has its own feature that UW is fixed and known to the receiver so it can be conveniently used to channel estimation as the training sequences[8].

On the ideal condition UW should have the feature of constant-amplitude in the frequency domain because of its function of channel estimation,so that it can produce relative stable frequency response to test the channel characteristics at frequency points. This simulation adopts the CAZAC sequences[9][10], which have a outstanding of the constant amplitude in time and frequency domain. The CAZAC sequences are defined as follow:

$$
\begin{gathered}
C(k)=\exp \left(2 \pi j\left(n+\frac{n(n+1)}{2}\right) / N, k=0,1,2, \ldots, N-1(N \text { is odd })\right. \\
C(k)=\exp \left(2 \pi j\left(n+\frac{n^{2}}{2}\right) / N, k=0,1,2, \ldots, N-1(N \text { is even })\right.
\end{gathered}
$$

But, the first UW for each frame absorbs the multipath interference from the previous data block,so if this UW is used to channel estimation, the accuracy will be reduced.Therefore, a special sequence named STS(Synchronization Training Sequence) is inserted for channel estimation, shows as in the Fig .3. The structure of STS is[A A], and A is a CAZAC sequence of 160 points.The first $A$ is used as guard interval and the second is used to channel estimation., so it has a better property of channel estimation. Now this frame structure is widely used in the SC-FDE and has good performance.

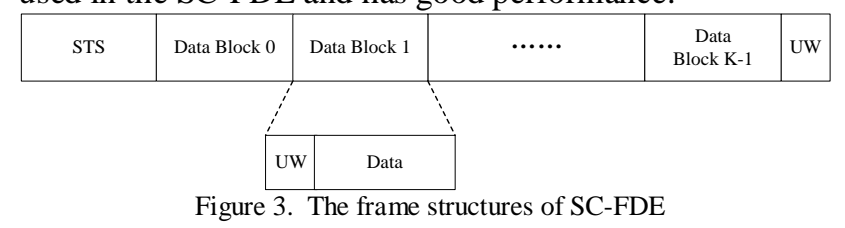

\section{B. Settings of Simulation Parameters}

For SC-FDE system simulation, sets the channel model for the non time varying rician channel, which contains four paths.Channel parameters are shown in Table I. Assuming the symbol rate is $8 \mathrm{KSps}$, the UW uses the CAZAC sequence of 64 points, and the total length of a data block is 320.Each frame contains 15 data blocks. Modulation mode can be selected, and convolution encoding is applied. The sending parameters of the simulation are shown in Table II.

TABLE I. ChanNel PARAMETERS

\begin{tabular}{|l|l|}
\hline Delay(ms) & Power(dB) \\
\hline 0 & 0 \\
\hline 0.75 & -3 \\
\hline 1.5 & -6 \\
\hline 2.25 & -9 \\
\hline
\end{tabular}

TABLE II. SENDING PARAMETERS

\begin{tabular}{|l|l|}
\hline \multicolumn{1}{|c|}{ Parameters } & \multicolumn{1}{c|}{ Values } \\
\hline Modulation Mode & QPSK/16QAM/64QAM \\
\hline Encoding Mode & Convolution Encoding \\
\hline Encoding Rate & 0.5 \\
\hline Length of STS & 320 \\
\hline Type of STS & CAZAC \\
\hline Length of UW(L) & 64 \\
\hline Type of UW & CAZAC \\
\hline Length of Data(M) & 256 \\
\hline Length of Data Block(P) & 320 \\
\hline
\end{tabular}


From the simulation of IBDFE[6],we note that the first two iterations yield a significant gain in performance, while the gain of further iterations is reduced. In the simulation results given below, the number of iterations for IBDFE is defined as $N_{I}=3$.

Based on the LS criterion, the channel frequency response of 160 points is obtained by using the second $\mathrm{A}$ of STS, then a 320 points frequency response is calculated by 2 times Wiener interpolation filtering. Due to the non time varying channel setting, that channel frequency response of each frame is unchanged. Fig .4 and Fig .5 shows the channel impulse response and constellations of different equalization schemes respectively, in the condition of QPSK, while SNR is 10dB.In Fig .5, the four constellations are corresponding to ZF,MMSE,DFE and IBDFE respectively, from left to right and from up to down.Similarly,Fig .6 shows the constellations using 16QAM,while SNR is 18dB. Fig .7 shows the constellations using 64QAM, while SNR is $25 \mathrm{~dB}$.

As can be seen, the performance of $\mathrm{ZF}$ is the worst, the performance of DFE and IBDFE is better. The performance of different schemes is analyzed and compared in detail in the next section.

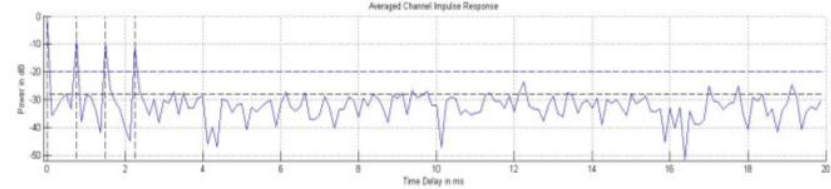

Figure 4. The channel impulse response
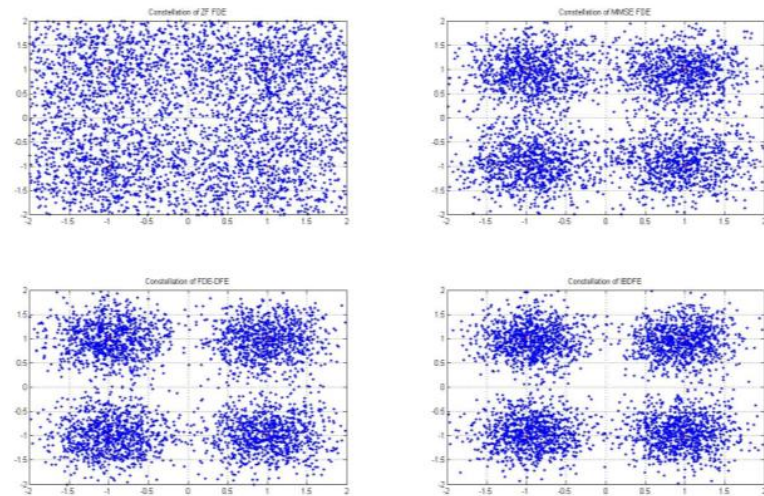

Figure 5. Constellations Comparison of QPSK
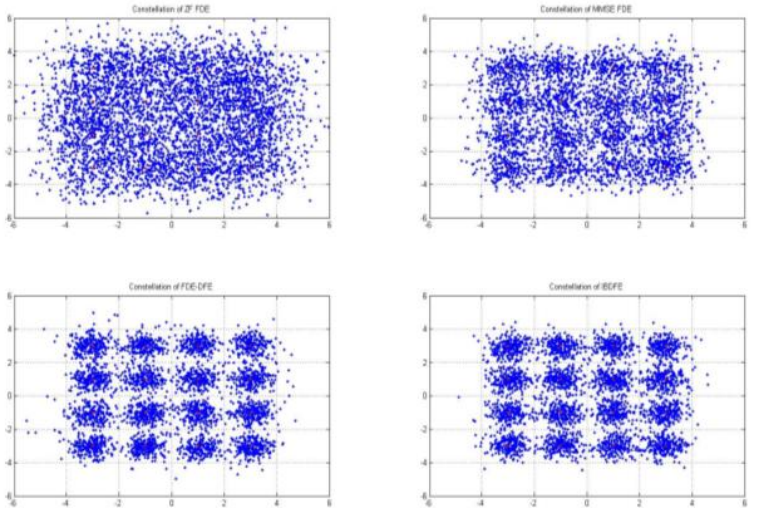

Figure 6. Constellations Comparison of 16QAM
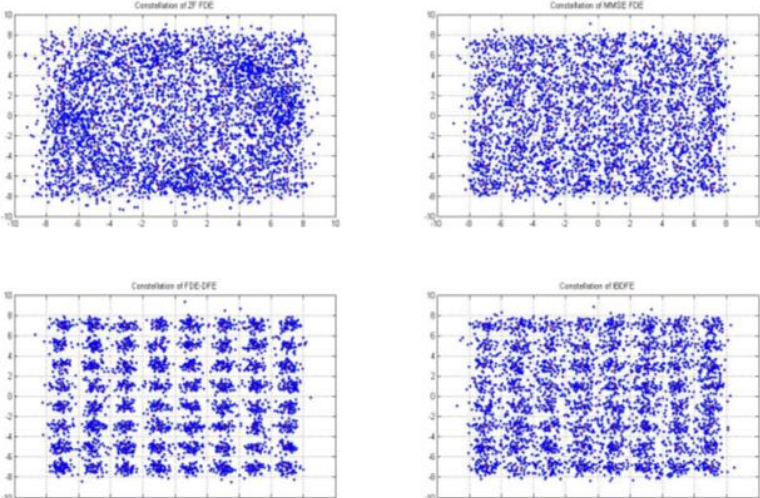

Figure 7. Constellations Comparison of 64QAM

\section{Performance Comparison}

Fig .8,Fig .9 and Fig .10 shows the BER of four equalization schemes, under the condition of QPSK,16QAM and 64QAM respectively.

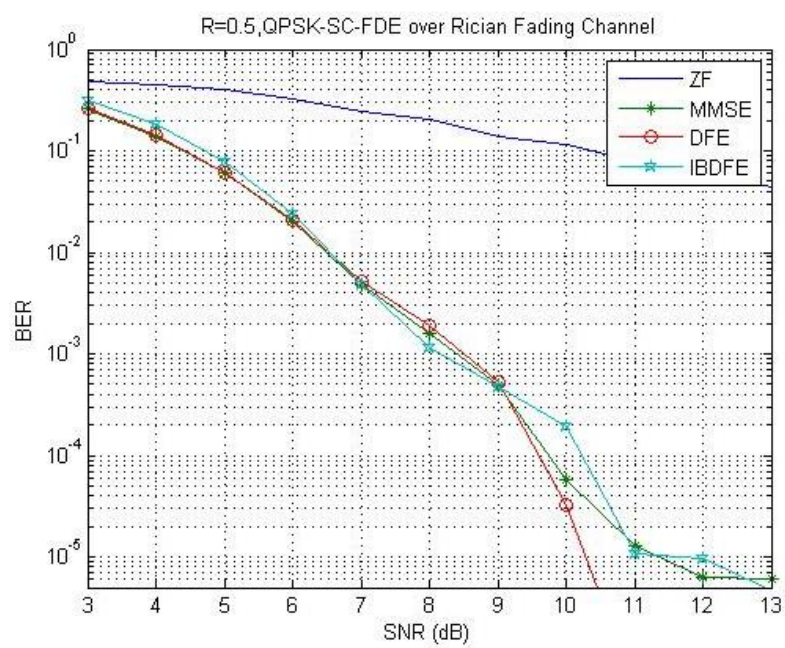

Figure 8. Performance Comparison of QPSK

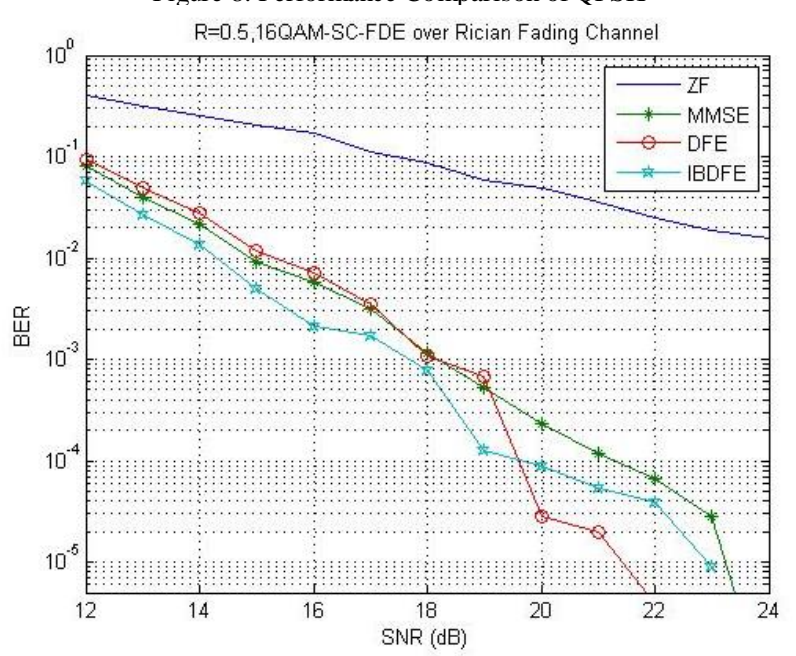

Figure 9. Performance Comparison of 16QAM 


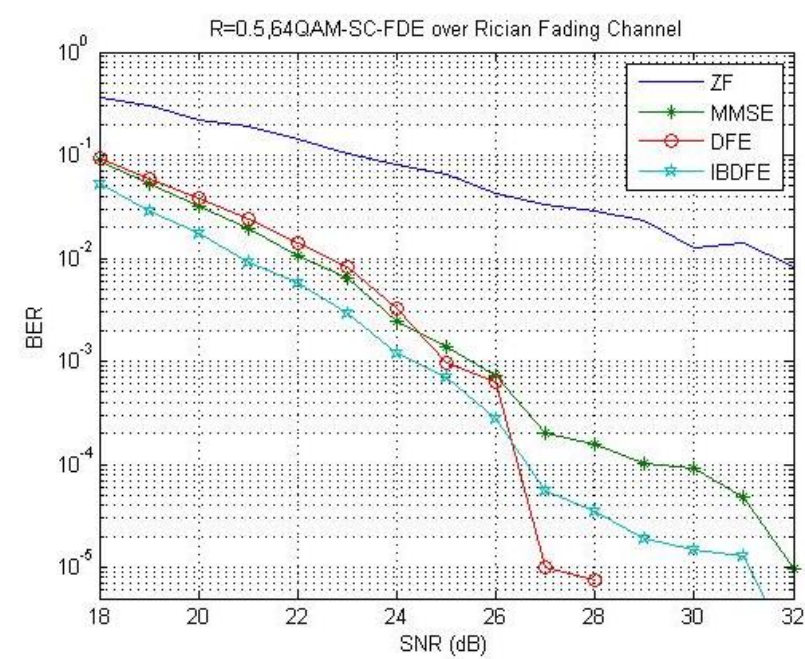

Figure 10. Performance Comparison of 64QAM

As can be seen, the $\mathrm{ZF}$ scheme is very sensitive to noise and frequency domain deep fading, and it is easy to amplify the noise, so it shows the worst BER performance. The MMSE scheme improves the performance,for the reason that it takes into account the ISI and noise, so that the ISI and noise can be suppressed. Due to the use of feedforward and feedback filters, the DFE and IBDFE could greatly decrease the influence of ISI and noise. Compared to the MMSE scheme, performance has greatly improved. Overall, the DFE is the best.

Under the condition of QPSK modulation, the required SNR difference among MMSE,DFE and IBDFE is less than $1 \mathrm{~dB}$ at $\mathrm{BER}=10^{-4}$. We note that the MMSE and IBDFE performs almost the same at $\mathrm{BER}=10^{-5}$. However, when compared with the MMSE and IBDFE, the DFE obtained a gain about $1 \mathrm{~dB}$ at $\mathrm{BER}=10^{-5}$.

Under the condition of 16QAM modulation, when compared with the MMSE, we note that the DFE and IBDFE yields a gain about $1.6 \mathrm{~dB}$ at $\mathrm{BER}=10^{-4}$. The IBDFE obtained $0.3 \mathrm{~dB}$ and the DFE obtained $1.8 \mathrm{~dB}$ at $\mathrm{BER}=10^{-5}$ approximately.

Under the condition of 64QAM modulation, we note that the DFE and IBDFE outperforms the MMSE by about $2.5 \mathrm{~dB}$ at $\mathrm{BER}=10^{-4}$. The IBDFE obtained $1 \mathrm{~dB}$ and the DFE yields a gain about $5 \mathrm{~dB}$ at $\mathrm{BER}=10^{-5}$ approximately.

\section{Computational Complexity}

The computational complexity of the various schemes is evaluated in terms of the number of complex multiplications(CMULs). We have considered that a $P$ size DFT requires $(P / 2) \log _{2}(P)-P$ CMULs, while the inversion of a correlation matrix of size $Q$ in the design of the DFE has a complexity of $\mathrm{O}\left(Q^{2}\right)$ [6]. In the comparisons for the IBDFE,we considered both $N_{I}=2$ and $N_{I}=3$. Table III shows the number of required CMULs for every $\mathrm{P}$ output samples of the equalizer design.
TABLE III.

COMPUTATIONAL COMPLEXITY

\begin{tabular}{|c|c|c|c|}
\hline Sche me & The number of CMULs & Simulation Result \\
\hline ZF & $2 P$ & \multicolumn{2}{|c|}{640} \\
\hline MMSE & $2 P$ & \multicolumn{2}{|c|}{640} \\
\hline DFE & $\mathrm{O}\left[(2 L)^{2}+2 P+P \log _{2} P\right]$ & \multicolumn{2}{|c|}{19687} \\
\hline IBDFE & $\left(3 N_{I}+1\right) P$ & $N_{I}=2$ & 2240 \\
& & $N_{I}=3$ & 3200 \\
\hline
\end{tabular}

In terms of numbers of CMULs, the complexity of DFE is the highest, and MMSE and ZF are the lowest. The complexity of IBDFE is the center, but it needs to calculate the signal statistics.

\section{CONCLUSION}

The signal processing method of SC-FDE is similar to OFDM,but it avoids the disadvantages of OFDM system with its good anti multipath capability and low complexity. This paper mainly focuses on the technology of single carrier frequency domain equalization in broadband HF channel. The characteristics of HF channel and the principle and implementation of SC-FDE system are introduced. The paper implements the system simulation and shows performance of different equalization schemes. The complexity of the schemes is evaluated in the end. In the practical engineering application, the appropriate equalization scheme can be selected according to the specific channel environment and communication requirements.

\section{ACKNOWLEDGMENT}

The work of this paper is supported by the Sponsors of 2012BAH17B01, National Science \& Technology Pillar Program.

\section{REFERENCES}

[1] David Falconer, Sirikiat Lek Ariyavisitakul, and Anader Benyamin-Seeyar, "Frequency Domain Equalization for SingleCarrier Broadband Wireless Systems[J]," IEEE Communications Magazine, Apr.2002, pp.58-66.

[2] M.Huemer, A. Koppler, and R.Weigel, "A review of cyclically extended single carrier transmission with frequency domain equliazation for broadband wirelesstransmission," Eruopean Transactions on Telecommunications, 2003, Vol.14, pp.329 341.

[3] Qing Zhang, and Tho Le-Ngoc, "Channel-estimate-based frequency-domain equalization (CEFDE)for broadband singlecarrier transmission," Wireless Communication and Mobile Computing, 2004(4), pp.449-461

[4] Martin V.Clark, "Adaptive Frequency-Domain Equalization and Diversity Combining for Broadband Wireless Communications[J]," IEEE Journal on Selected Areas in Communications, Oct.1998, Vol.16, pp.1385-1390

[5] Benvenuto N, and Tomasin S, "On the comparison between OFDM and single carrier modulation with a DFE using a frequencydomain feedforward filter[J]," IEEE Transactions on Communications, 2002, 50(6), pp.947-955.

[6] Benvenuto N, and Tomasin S, "Iterative design and detection of a DFE in the frequency domain[J]," IEEE Transactions on Communications, 2005, 53(11), pp. 1867-1875.

[7] David C.Chu, "Polyphase Codes With Good Periodic Correlation Properties," IEEE Transactions on Information theory, Jul.1972, pp.531-532.

[8] Mario Huemer, Harald Witschnig, and Josef Hausner, "Unique word based phase tracking algorithms for SC/FDE-systems," Global telecommunications Conference, Dec.2003, Vol.1,pp.70-74. 
[9] R.L.Frank, and S.A.Zadoff, "Phase Shift Pulse Codes With Good Periodic Correlation Properties," IRE Transactions on Information theory, Oct.1962, pp.381-382.
[10] David C.Chu, "Polyphase Codes With Good Periodic Correlation Properties," IEEE Transactions on Information theory, Jul.1972, pp.531-532. 